\title{
A regularity criterion for the generalized Hall-MHD system
}

\author{
Weijiang Gu', Caochuan Ma²,3* and Jianzhu Sun ${ }^{4}$
}

"Correspondence:
ccma@zjnu.edu.cn
2Department of Mathematics,
Tianshui Normal University,
Tianshui, P.R. China
${ }^{3}$ Department of Mathematics,
Zhejiang Normal University, Jinhua,
P.R. China
Full list of author information is
available at the end of the article

available at the end of the article

\author{
Abstract \\ This paper proves a regularity criterion for the 3D generalized Hall-MHD system. \\ MSC: 35Q35; 35B65; 76D05 \\ Keywords: regularity criterion; generalized Hall-MHD; Gagliardo-Nirenberg \\ inequality
}

\section{Introduction}

In this paper, we consider the following 3D generalized Hall-MHD system:

$$
\begin{aligned}
& \partial_{t} u+u \cdot \nabla u+\nabla\left(\pi+\frac{1}{2}|b|^{2}\right)+(-\Delta)^{\alpha} u=b \cdot \nabla b, \\
& \partial_{t} b+u \cdot \nabla b-b \cdot \nabla u+(-\Delta)^{\beta} b+\operatorname{rot}(\operatorname{rot} b \times b)=0, \\
& \operatorname{div} u=\operatorname{div} b=0, \\
& (u, b)(\cdot, 0)=\left(u_{0}, b_{0}\right) \quad \text { in } \mathbb{R}^{3} .
\end{aligned}
$$

Here $u, \pi$, and $b$ denote the velocity, pressure, and magnetic field of the fluid, respectively. $0<\alpha, \beta$ are two constants. The fractional Laplace operator $(-\Delta)^{\alpha}$ is defined through the Fourier transform, namely, $\widehat{(-\Delta)^{\alpha} f}(\xi)=|\xi|^{2 \alpha} \hat{f}(\xi)$.

The applications of the Hall-MHD system cover a very wide range of physical objects, for example, magnetic reconnection in space plasmas, star formation, neutron stars, and the geo-dynamo.

When the Hall effect term $\operatorname{rot}(\operatorname{rot} b \times b)$ is neglected, the system (1.1)-(1.4) reduces to the well-known generalized MHD system, which has received attention in many studies [1-6].

When $\alpha=\beta=1$, the system (1.1)-(1.4) reduces to the well-known Hall-MHD system, which has received many studies [7-11]. Reference [7] gave a derivation of the isentropic Hall-MHD system from a two-fluid Euler-Maxwell system. Chae-Degond-Liu [8] proved the local existence of smooth solutions. Chae and Schonbek [9] showed the time-decay and some regularity criteria were proved in [10-12]. Some other relevant results about Hall-MHD equations can be found in [13-17].

The local well-posedness is established in Wan and Zhou [18] when $0<\alpha \leq 1$ and $\frac{1}{2}<$ $\beta \leq 1$.

(c) 2016 Gu et al. This article is distributed under the terms of the Creative Commons Attribution 4.0 International License (http://creativecommons.org/licenses/by/4.0/), which permits unrestricted use, distribution, and reproduction in any medium, provided you give appropriate credit to the original author(s) and the source, provide a link to the Creative Commons license, and indicate if changes were made. 
When $\frac{3}{4} \leq \alpha<\frac{5}{4}$ and $1 \leq \beta<\frac{7}{4}$, Jiang and Zhu [19] prove the following regularity criteria:

$$
\nabla b \in L^{t}\left(0, T ; L^{s}\right) \quad \text { with } \frac{2 \beta}{t}+\frac{3}{s} \leq 2 \beta-1, \frac{3}{2 \beta-1}<s \leq \infty,
$$

and one of the following two conditions:

$$
u \in L^{p}\left(0, T ; L^{q}\right) \quad \text { with } \frac{2 \alpha}{p}+\frac{3}{q} \leq 2 \alpha-1, \frac{3}{2 \alpha-1}<q \leq \frac{6 \alpha}{2 \alpha-1},
$$

or

$$
\Lambda^{\alpha} u \in L^{p}\left(0, T ; L^{q}\right) \quad \text { with } \frac{2 \alpha}{p}+\frac{3}{q} \leq 3 \alpha-1, \frac{3}{3 \alpha-1}<q \leq \frac{6 \alpha}{3 \alpha-1} .
$$

When $1 \leq \alpha<\frac{5}{4}$ and $1 \leq \beta<\frac{7}{4}$, Ye [20] showed the following regularity criterion:

$$
u \in L^{p}\left(0, T ; L^{q}\right) \text { and } \nabla b \in L^{\ell}\left(0, T ; L^{k}\right)
$$

where $p, q, \ell$, and $k$ satisfy the relation

$$
\frac{3}{q}+\frac{2 \alpha}{p} \leq 2 \alpha-1, \quad \frac{3}{q}+\frac{2 \beta}{p} \leq 2 \beta-1, \quad \frac{3}{k}+\frac{2 \beta}{\ell} \leq 2 \beta-1,
$$

and

$$
\max \left(\frac{3}{2 \alpha-1}, \frac{3}{2 \beta-1}\right)<q \leq \infty, \quad \frac{3}{2 \beta-1}<k \leq \infty .
$$

The aim of this paper is to refine the results in $[19,20]$ as follows.

Theorem 1.1 Let $\frac{3}{4} \leq \alpha<\frac{5}{4}$ and $\frac{3}{4} \leq \beta<\frac{7}{4}$ and $u_{0}, b_{0} \in H^{2}$ with $\operatorname{div} u_{0}=\operatorname{div} b_{0}=0$ in $\mathbb{R}^{3}$. If $\nabla u$ and $\nabla$ satisfy

$$
\begin{aligned}
& \nabla u \in L^{\frac{2 \alpha}{2 \alpha-\gamma_{1}}}\left(0, T ; \dot{B}_{\infty, \infty}^{-\gamma_{1}}\right), \\
& \nabla b \in L^{\frac{2 \beta}{2 \beta-1-\gamma_{2}}}\left(0, T ; \dot{B}_{\infty, \infty}^{-\gamma_{2}}\right) \quad \text { with } 0<\gamma_{1}<2 \alpha \text { and } 0<\gamma_{2}<2 \beta-1,
\end{aligned}
$$

with $0<T<\infty$, then the solution $(u, b)$ can be extended beyond $T$.

In the following proof, we will use the following bilinear products and commutator estimates due to Kato-Ponce [21]:

$$
\begin{aligned}
& \left\|\Lambda^{s}(f g)\right\|_{L^{p}} \leq C\left(\left\|\Lambda^{s} f\right\|_{L^{p_{1}}}\|g\|_{L^{q_{1}}}+\|f\|_{L^{p_{2}}}\left\|\Lambda^{s} g\right\|_{L^{q_{2}}}\right), \\
& \left\|\Lambda^{s}(f g)-f \Lambda^{s} g\right\|_{L^{p}} \leq C\left(\|\nabla f\|_{L^{p_{1}}}\left\|\Lambda^{s-1} g\right\|_{L^{q_{1}}}+\left\|\Lambda^{s} f\right\|_{L^{p_{2}}}\|g\|_{L^{q_{2}}}\right),
\end{aligned}
$$

with $s>0, \Lambda:=(-\Delta)^{\frac{1}{2}}$, and $\frac{1}{p}=\frac{1}{p_{1}}+\frac{1}{q_{1}}=\frac{1}{p_{2}}+\frac{1}{q_{2}}$.

We will also use the improved Gagliardo-Nirenberg inequalities [22-24]:

$$
\begin{aligned}
& \|\nabla u\|_{L^{3}}^{3} \leq C\|\nabla u\|_{\dot{B}_{\infty, \infty}^{-\gamma_{1}}}\|\nabla u\|_{\dot{H}^{\frac{\gamma_{1}}{2}}}^{2}, \\
& \|\nabla b\|_{L^{3}}^{3} \leq C\|\nabla b\|_{\dot{B}_{\infty, \infty}^{-\gamma_{2}}}\|\nabla b\|_{\dot{H}^{\frac{\gamma_{2}}{2}}}^{2},
\end{aligned}
$$




$$
\begin{aligned}
& \|\nabla b\|_{L^{p_{3}}} \leq C\|\nabla b\|_{\dot{B}_{\infty, \infty}^{-\gamma \gamma_{2}}}^{1-\theta_{1}}\|\nabla b\|_{\dot{H}^{s}}^{\theta_{1}}, \\
& \left\|\Lambda^{2-\beta} b\right\|_{L^{q_{3}}} \leq C\|\nabla b\|_{\dot{B}_{\infty, \infty}^{-\gamma_{2}}}^{\theta_{1}}\|\nabla b\|_{\dot{H}^{s}}^{\theta_{1}},
\end{aligned}
$$

with $\theta_{1}:=\frac{\gamma_{2}}{s+\gamma_{2}}, s:=1+\gamma_{2}-\beta, p_{3}:=\frac{2}{\theta_{1}}$ and $q_{3}:=\frac{2}{1-\theta_{1}}$

$$
\begin{aligned}
& \|\nabla b\|_{L^{p_{4}}} \leq C\|\nabla b\|_{\dot{B}_{\infty, \infty}^{-\gamma_{2}}}^{1-\theta_{2}}\|\Delta b\|_{\dot{H}^{s}}^{\theta_{2}}, \\
& \left\|\Lambda^{3-\beta} b\right\|_{L^{q_{4}}} \leq C\|\nabla b\|_{\dot{B}_{\infty, \infty}^{-\gamma_{2}}}^{\theta_{2}}\|\Delta b\|_{H^{s}}^{1-\theta_{2}}
\end{aligned}
$$

with $\theta_{2}:=\frac{\gamma_{2}}{1+s+\gamma_{2}}, p_{4}:=\frac{2}{\theta_{2}}$, and $q_{4}:=\frac{2}{1-\theta_{2}}$. We have

$$
\begin{gathered}
\left\|\Lambda^{2-\beta} b\right\|_{L^{p_{5}}} \leq C\|\nabla b\|_{\dot{B}_{\infty, \infty}^{-\gamma_{2}}}^{1-\theta_{2}}\|\Delta b\|_{\dot{H}^{s}}^{\theta_{3}}, \\
\|\Delta b\|_{L^{q_{5}}} \leq C\|\nabla b\|_{\dot{B}_{\infty, \infty}^{-\gamma_{2}}}^{\theta_{3}}\|\Delta b\|_{\dot{H}^{s}}^{1-\theta_{3}},
\end{gathered}
$$

with $\theta_{3}:=\frac{s}{1+s+\gamma_{2}}, p_{5}:=\frac{2}{\theta_{3}}$, and $q_{5}:=\frac{2}{1-\theta_{3}}$.

\section{Proof of Theorem 1.1}

This section is devoted to the proof of Theorem 1.1, we only need to prove a priori estimates.

First, testing (1.1) by $u$ and using (1.3), we see that

$$
\frac{1}{2} \frac{d}{d t} \int|u|^{2} d x+\int\left|\Lambda^{\alpha} u\right|^{2} d x=\int(b \cdot \nabla) b \cdot u d x
$$

Testing (1.2) by $b$ and using (1.3), we find that

$$
\frac{1}{2} \frac{d}{d t} \int|b|^{2} d x+\int\left|\Lambda^{\beta} b\right|^{2} d x=\int(b \cdot \nabla) u \cdot b d x
$$

Summing up the above two equations, we get the well-known energy inequality

$$
\frac{1}{2} \int\left(|u|^{2}+|b|^{2}\right) d x+\int_{0}^{T} \int\left(\left|\Lambda^{\alpha} u\right|^{2}+\left|\Lambda^{\beta} b\right|^{2}\right) d x d t \leq \frac{1}{2} \int\left(\left|u_{0}\right|^{2}+\left|b_{0}\right|^{2}\right) d x
$$

Testing (1.1) by $-\Delta u$ and using (1.3), we infer that

$$
\begin{aligned}
& \frac{1}{2} \frac{d}{d t} \int|\nabla u|^{2} d x+\int\left|\Lambda^{1+\alpha} u\right|^{2} d x \\
& \quad=\int u \cdot \nabla u \cdot \Delta u d x-\int b \cdot \nabla b \cdot \Delta u d x \\
& \quad=-\sum_{i, j} \int \partial_{j} u_{i} \partial_{i} u \partial_{j} u d x+\sum_{i, j} \int \partial_{j} b_{i} \partial_{i} b \partial_{j} u d x+\sum_{i, j} \int b_{i} \partial_{i} \partial_{j} b \partial_{j} u d x \\
& \quad \leq C\|\nabla u\|_{L^{3}}^{3}+C\|\nabla b\|_{L^{3}}^{3}+\sum_{i, j} \int b_{i} \partial_{i} \partial_{j} b \partial_{j} u d x=: I_{1}+I_{2}+I_{3} .
\end{aligned}
$$


Testing (1.2) by $-\Delta b$ and using (1.3), we deduce that

$$
\begin{aligned}
& \frac{1}{2} \frac{d}{d t} \int|\nabla b|^{2} d x+\int\left|\Lambda^{1+\beta} b\right|^{2} d x \\
& =\int u \cdot \nabla b \cdot \Delta b d x-\int b \cdot \nabla u \cdot \Delta b d x+\int(\operatorname{rot} b \times b) \Delta \operatorname{rot} b d x \\
& =-\sum_{i, j} \int \partial_{j} u_{i} \partial_{i} b \partial_{j} b d x+\sum_{i, j} \int \partial_{j} b_{i} \partial_{i} u \partial_{j} b d x \\
& \quad+\sum_{i, j} \int b_{i} \partial_{i} \partial_{j} u \partial_{j} b d x-\sum_{i} \int\left(\operatorname{rot} b \times \partial_{i} b\right) \partial_{i} \operatorname{rot} b d x \\
& \leq C\|\nabla u\|_{L^{3}}^{3}+C\|\nabla b\|_{L^{3}}^{3}+\sum_{i, j} \int b_{i} \partial_{i} \partial_{j} u \partial_{j} b d x-\sum_{i} \int\left(\operatorname{rot} b \times \partial_{i} b\right) \partial_{i} \operatorname{rot} b d x \\
& =: I_{1}+I_{2}+I_{4}+I_{5} .
\end{aligned}
$$

Summing up (2.2) and (2.3), using (1.6), (1.8), (1.9), (1.10), (1.11), and $I_{3}+I_{4}=0$, we derive

$$
\begin{aligned}
& \frac{1}{2} \frac{d}{d t} \int\left(|\nabla u|^{2}+|\nabla b|^{2}\right) d x+\int\left(\left|\Lambda^{1+\alpha} u\right|^{2}+\left|\Lambda^{1+\beta} b\right|^{2}\right) d x \\
& \leq C\|\nabla u\|_{L^{3}}^{3}+C\|\nabla b\|_{L^{3}}^{3}-\sum_{i} \int \Lambda^{1-\beta}\left(\operatorname{rot} b \times \partial_{i} b\right) \cdot \Lambda^{\beta-1} \partial_{i} \operatorname{rot} b d x \\
& \leq C\|\nabla u\|_{L^{3}}^{3}+C\|\nabla b\|_{L^{3}}^{3}+C\|\nabla b\|_{L^{p_{3}}}\left\|\Lambda^{2-\beta} b\right\|_{L^{q 3}}\left\|\Lambda^{1+\beta} b\right\|_{L^{2}} \\
& \leq C\|\nabla u\|_{\dot{B}_{\infty, \infty}^{-\gamma_{1}}}\|\nabla u\|_{\dot{H}^{\frac{\gamma_{1}}{2}}}^{2}+C\|\nabla b\|_{\dot{B}_{\infty, \infty}^{-\gamma_{2}}}\|\nabla b\|_{\dot{H}^{\frac{\gamma_{2}}{2}}}^{2}+C\|\nabla b\|_{\dot{B}_{\infty, \infty}^{-\gamma_{2}}}\|\nabla b\|_{\dot{H}^{s}}\left\|\Lambda^{1+\beta} b\right\|_{L^{2}} \\
& \leq C\|\nabla u\|_{\dot{B}_{\infty, \infty}^{-\gamma_{1}}}\|\nabla u\|_{L^{2}}^{2\left(1-\frac{\gamma_{1}}{2 \alpha}\right)}\left\|\Lambda^{1+\alpha} u\right\|_{L^{2}}^{2 \cdot \frac{\gamma_{1}}{2 \alpha}}+C\|\nabla b\|_{\dot{B}_{\infty, \infty}^{-\gamma_{2}}}\|\nabla b\|_{L^{2}}^{2\left(1-\frac{\gamma_{2}}{2 \beta}\right)}\left\|\Lambda^{1+\beta} b\right\|_{L^{2}}^{2 \cdot \frac{\gamma_{2}}{2 \beta}} \\
& \quad+C\|\nabla b\|_{\dot{B}_{\infty, \infty}^{-\gamma_{2}}}\|\nabla b\|_{L^{2}}^{1-\frac{s}{\beta}}\left\|\Lambda^{1+\beta} b\right\|_{L^{2}}^{1+\frac{s}{\beta}} \\
& \leq \frac{1}{2}\left\|\Lambda \Lambda^{1+\alpha} u\right\|_{L^{2}}^{2}+\frac{1}{2}\left\|\Lambda^{1+\beta} b\right\|_{L^{2}}^{2}+C\|\nabla u\|_{\dot{B}_{\infty, \infty}^{-\gamma_{1}}}^{2 \alpha-\gamma_{1}}\|\nabla u\|_{L^{2}}^{2} \\
&+C\|\nabla b\|_{\dot{B}_{\infty, \infty}^{-\gamma_{2}}}^{\frac{2 \beta}{2 \beta-\gamma_{2}}}\|\nabla b\|_{L^{2}}^{2}+C\|\nabla b\|_{\dot{B}_{\infty, \infty}^{-\gamma_{2}}}^{\frac{2 \beta}{2 \beta-\gamma_{2}}}\|\nabla b\|_{L^{2}}^{2}
\end{aligned}
$$

which gives

$$
\|(u, b)\|_{L^{\infty}\left(0, T ; H^{1}\right)}+\|u\|_{L^{2}\left(0, T ; H^{1+\alpha}\right)}+\|b\|_{L^{2}\left(0, T ; H^{1+\beta}\right)} \leq C .
$$

In the following proofs, we will use the following Sobolev embedding theorem:

$$
\begin{aligned}
\|\nabla u\|_{L^{4}} \leq C\|u\|_{H^{1+\alpha}}, & \|\Delta u\|_{L^{4}} \leq C\|u\|_{H^{2+\alpha}} \leq C\left\|\Lambda^{2+\alpha} u\right\|_{L^{2}}+C, \\
\|\nabla b\|_{L^{4}} \leq C\|b\|_{H^{1+\beta}}, & \|\Delta b\|_{L^{4}} \leq C\left\|\Lambda^{2+\beta} b\right\|_{L^{2}}+C .
\end{aligned}
$$

Taking $\Delta$ to (1.1), testing by $\Delta u$ and using (1.3), we have

$$
\begin{aligned}
& \frac{1}{2} \frac{d}{d t} \int|\Delta u|^{2} d x+\int\left|\Lambda^{2+\alpha} u\right|^{2} d x \\
& \quad=-\int(\Delta(u \cdot \nabla u)-u \cdot \nabla \Delta u) \Delta u d x+\int(\Delta(b \cdot \nabla b)-b \cdot \nabla \Delta b) \Delta u d x
\end{aligned}
$$




$$
\begin{aligned}
& +\int b \cdot \nabla \Delta b \cdot \Delta u d x \\
=: & I_{6}+I_{7}+I_{8} .
\end{aligned}
$$

Applying $\Delta$ to (1.2), testing by $\Delta b$ and using (1.3), we have

$$
\begin{aligned}
\frac{1}{2} \frac{d}{d t} \int|\Delta b|^{2} d x+\int\left|\Lambda^{2+\beta} b\right|^{2} d x \\
=-\int(\Delta(u \cdot \nabla b)-u \cdot \nabla \Delta b) \Delta b d x+\int(\Delta(b \cdot \nabla u)-b \cdot \nabla \Delta u) \Delta b d x \\
\quad+\int b \cdot \nabla \Delta u \cdot \Delta b d x-\int \Delta(\operatorname{rot} b \times b) \cdot \Delta \operatorname{rot} b d x \\
=: I_{9}+I_{10}+I_{11}+I_{12} .
\end{aligned}
$$

Note that $I_{8}+I_{11}=0$.

Using (1.7), (2.4), and (2.5), we bound $I_{6}+I_{7}+I_{9}+I_{10}$ as follows:

$$
\begin{aligned}
I_{6}+ & I_{7}+I_{9}+I_{10} \\
\leq & C\|\nabla u\|_{L^{4}}\|\Delta u\|_{L^{4}}\|\Delta u\|_{L^{2}}+C\|\nabla b\|_{L^{4}}\|\Delta b\|_{L^{4}}\|\Delta u\|_{L^{2}} \\
& +C\left(\|\nabla b\|_{L^{4}}\|\Delta u\|_{L^{4}}+\|\nabla u\|_{L^{4}}\|\Delta b\|_{L^{4}}\right)\|\Delta b\|_{L^{2}} \\
\leq & C\|u\|_{H^{1+\alpha}}\|u\|_{H^{2+\alpha}}\|\Delta u\|_{L^{2}}+C\|b\|_{H^{1+\beta}}\|b\|_{H^{2+\beta}}\|\Delta u\|_{L^{2}} \\
& +C\left(\|b\|_{H^{1+\beta}}\|u\|_{H^{2+\alpha}}+\|u\|_{H^{1+\alpha}}\|b\|_{H^{2+\beta}}\right)\|\Delta b\|_{L^{2}} \\
& +\frac{1}{8}\|u\|_{H^{2+\alpha}}^{2}+\frac{1}{8}\|b\|_{H^{2+\beta}}^{2}+C\left(\|u\|_{H^{1+\alpha}}^{2}+\|b\|_{H^{1+\beta}}^{2}\right)\left(\|\Delta u\|_{L^{2}}^{2}+\|\Delta b\|_{L^{2}}^{2}\right) .
\end{aligned}
$$

Using (1.6), (1.12), (1.13), (1.14), and (1.15), we bound $I_{12}$ as follows:

$$
\begin{aligned}
I_{12}= & -\int(\operatorname{rot} b \times \Delta b) \cdot \Delta \operatorname{rot} b d x-2 \sum_{i} \int\left(\partial_{i} \operatorname{rot} b \times \partial_{i} b\right) \Delta \operatorname{rot} b d x \\
= & -\int \Lambda^{1-\beta}(\operatorname{rot} b \times \Delta b) \cdot \Lambda^{\beta-1} \Delta \operatorname{rot} b d x \\
& -2 \sum_{i} \int \Lambda^{1-\beta}\left(\partial_{i} \operatorname{rot} b \times \partial_{i} b\right) \cdot \Lambda^{\beta-1} \Delta \operatorname{rot} b d x \\
\leq & C\left(\|\nabla b\|_{L^{p_{4}}}\left\|\Lambda^{3-\beta} b\right\|_{L^{q 4}}+\left\|\Lambda^{2-\beta} b\right\|_{L^{p_{5}}}\|\Delta b\|_{L^{q}}\right)\left\|\Lambda^{2+\beta} b\right\|_{L^{2}} \\
\leq & C\|\nabla b\|_{\dot{B}_{\infty, \infty}^{-\gamma_{2}}}\|\Delta b\|_{\dot{H}^{s}}\left\|\Lambda^{2+\beta} b\right\|_{L^{2}} \\
\leq & C\|\nabla b\|_{\dot{B}_{\infty, \infty}^{-\gamma_{2}}}\|\Delta b\|_{L^{2}}^{1-\frac{s}{\beta}}\left\|\Lambda^{2+\beta} b\right\|_{L^{2}}^{1+\frac{s}{\beta}} \\
\leq & \frac{1}{8}\left\|\Lambda^{2+\beta} b\right\|_{L^{2}}^{2}+C\|\nabla b\|_{\dot{B}_{\infty, \infty}^{-\gamma_{2}}}^{\frac{2 \beta}{2 \beta-\gamma_{2}}}\|\Delta b\|_{L^{2}}^{2} .
\end{aligned}
$$

Inserting the above estimates into (2.6) and (2.7), and summing up the results and using the Gronwall inequality, we arrive at

$$
\|(u, b)\|_{L^{\infty}\left(0, T ; H^{2}\right)}+\|u\|_{L^{2}\left(0, T ; H^{2+\alpha}\right)}+\|b\|_{L^{2}\left(0, T ; H^{2+\beta}\right)} \leq C .
$$

This completes the proof. 


\section{Conclusions}

The applications of Hall-MHD system cover a very wide range of physical objects, such as magnetic reconnection in space plasmas, star formation, neutron stars, and the geodynamo. In this paper, we obtained a new regularity criterion that improves and extends some known regularity criteria of the 3D generalized Hall-MHD system.

\section{Competing interests}

The authors declare that they have no competing interests.

\section{Authors' contributions}

All authors contributed equally to the writing of this paper. All authors read and approved the final manuscript.

\section{Author details}

${ }^{1}$ Center for Information, Nanjing Forestry University, Nanjing, 210037, P.R. China. ${ }^{2}$ Department of Mathematics, Tianshui Normal University, Tianshui, P.R. China. ${ }^{3}$ Department of Mathematics, Zhejiang Normal University, Jinhua, P.R. China. ${ }^{4}$ Department of Applied Mathematics, Nanjing Forestry University, Nanjing, 210037, P.R. China.

\section{Acknowledgements}

The authors would like to thank the anonymous referees and the editors for their valuable suggestions and comments, which improved the results and the quality of the paper. Ma is partially supported by NSFC (Grant No. 11661070), the Youth Science and Technology Support Program (Grant No. 1606RJYE237) and the Scientific Research Foundation of the Higher Education Institutions (Grant No. 2015A-131) of Gansu Province.

Received: 29 August 2016 Accepted: 13 October 2016 Published online: 24 October 2016

\section{References}

1. Fan, J, Gao, H, Nakamura, G: Regularity criteria for the generalized magnetohydrodynamic equations and the quasi-geostrophic equations. Taiwan. J. Math. 15, 1059-1073 (2011)

2. Wu, J: Generalized MHD equations. J. Differ. Equ. 195, 284-312 (2003)

3. Wu, J: Regularity criteria for the generalized MHD equatins. Commun. Partial Differ. Equ. 33, 285-306 (2008)

4. Zhou, Y: Regularity criteria for the generalized viscous MHD equations. Ann. Inst. Henri Poincaré, Anal. Non Linéaire 24, 491-505 (2007)

5. Fan, J, Malaikah, H, Monaquel, S, Nakamura, G, Zhou, Y: Global Cauchy problem of 2D generalized MHD equations. Monatshefte Math. 175(1), 127-131 (2014)

6. Jiang, Z, Wang, Y, Zhou, Y: On regularity criteria for the 2D generalized MHD system. J. Math. Fluid Mech. 18(2), 331-341 (2016)

7. Acheritogaray, M, Degond, P, Frouvelle, A, Liu, J: Kinetic formulation and global existence for the Hall-magnetohydrodynamics system. Kinet. Relat. Models 4, 901-918 (2011)

8. Chae, D, Degond, P, Liu, J: Well-posedness for Hall-magnetohydrodynamics. Ann. Inst. Henri Poincaré, Anal. Non Linéaire 31(3), 555-565 (2014)

9. Chae, D, Schonbek, M: On the temporal decay for the Hall-magnetohydrodynamic equations. J. Differ. Equ. 255(11), 3971-3982 (2013)

10. Wan, R, Zhou, Y: On the global existence, energy decay and blow up criterions for the Hall-MHD system. J. Differ. Equ. 259, 5982-6008 (2015)

11. Zhang, Z: A remark on the blow-up criterion for the 3D Hall-MHD system in Besov spaces. Preprint (2015)

12. Pan, N, Ma, C, Zhu, M: Global regularity for the 3D generalized Hall-MHD system. Appl. Math. Lett. 61, 62-66 (2016)

13. Fan, J, Alsaedi, A, Hayat, T, Nakamura, G, Zhou, Y: On strong solutions to the compressible Hall-magnetohydrodynamic system. Nonlinear Anal., Real World Appl. 22, 423-434 (2015)

14. Fan, J, Jia, X, Nakamura, G, Zhou, Y: On well-posedness and blowup criteria for the magnetohydrodynamics with the Hall and ion-slip effects. Z. Angew. Math. Phys. 66(4), 1695-1706 (2015)

15. Fan, J, Ahmad, B, Hayat, T, Zhou, Y: On blow-up criteria for a new Hall-MHD system. Appl. Math. Comput. 274, 20-24 (2016)

16. Fan, J, Ahmad, B, Hayat, T, Zhou, Y: On well-posedness and blow-up for the full compressible Hall-MHD system. Nonlinear Anal., Real World Appl. 31, 569-579 (2016)

17. He, F, Ahmad, B, Hayat, T, Zhou, Y: On regularity criteria for the 3D Hall-MHD equations in terms of the velocity. Nonlinear Anal., Real World Appl. 32, 35-51 (2016)

18. Wan, R, Zhou, Y: Low regularity well-posedness for the 3D generalized Hall-MHD system. Acta Appl. Math. (2016). doi:10.1007/s10440-016-0070-5

19. Jiang, Z, Zhu, M: Regularity criteria for the $3 D$ generalized MHD and Hall-MHD systems. Bull. Malays. Math. Soc. (2015). doi:10.1007/s40840-015-0243-9

20. Ye, Z: Regularity criteria and small data global existence to the generalized viscous Hall-magnetohydrodynamics. Comput. Math. Appl. 70, 2137-2154 (2015)

21. Kato, T, Ponce, G: Commutator estimates and the Euler and Navier-Stokes equations. Commun. Pure Appl. Math. 41, 891-907 (1988)

22. Hajaiej, H, Molinet, L, Ozawa, T, Wang, B: Necessary and sufficient conditions for the fractional Gagliardo-Nirenberg inequalities and applications to Navier-Stokes and generalized Boson equations. RIMS Kôkyûroku Bessatsu 26, 159-175 (2011)

23. Machihara, S, Ozawa, T: Interpolation inequalities in Besov spaces. Proc. Am. Math. Soc. 131, 1553-1556 (2002) 
24. Meyer, Y: Oscillation patterns in some nonlinear evolution equations. In: Cannone, M, Miyakawa, $T$ (eds.) Mathematical Foundation of Turbulent Viscous Flows. Lecture Notes in Mathematics, vol. 1871, pp. 101-187. Springer, Berlin (2006)

Submit your manuscript to a SpringerOpen ${ }^{\circ}$ journal and benefit from:

- Convenient online submission

Rigorous peer review

- Immediate publication on acceptance

- Open access: articles freely available online

- High visibility within the field

- Retaining the copyright to your article

Submit your next manuscript at $\boldsymbol{s p r i n g e r o p e n . c o m ~}$ 